PROCEEDINGS OF THE

AMERICAN MATHEMATICAL SOCIETY

Volume 125, Number 3, March 1997, Pages 731-738

S 0002-9939(97)03815-X

\title{
COMMENSURATORS OF PARABOLIC SUBGROUPS OF COXETER GROUPS
}

\author{
LUIS PARIS
}

(Communicated by Ronald M. Solomon)

\begin{abstract}
Let $(W, S)$ be a Coxeter system, and let $X$ be a subset of $S$. The subgroup of $W$ generated by $X$ is denoted by $W_{X}$ and is called a parabolic subgroup. We give the precise definition of the commensurator of a subgroup in a group. In particular, the commensurator of $W_{X}$ in $W$ is the subgroup of $w$ in $W$ such that $w W_{X} w^{-1} \cap W_{X}$ has finite index in both $W_{X}$ and $w W_{X} w^{-1}$. The subgroup $W_{X}$ can be decomposed in the form $W_{X}=W_{X^{0}} \cdot W_{X} \infty \simeq$ $W_{X^{0}} \times W_{X^{\infty}}$ where $W_{X^{0}}$ is finite and all the irreducible components of $W_{X^{\infty}}$ are infinite. Let $Y^{\infty}$ be the set of $t$ in $S$ such that $m_{s, t}=2$ for all $s \in X^{\infty}$. We prove that the commensurator of $W_{X}$ is $W_{Y^{\infty}} \cdot W_{X^{\infty}} \simeq W_{Y^{\infty}} \times W_{X \infty}$. In particular, the commensurator of a parabolic subgroup is a parabolic subgroup, and $W_{X}$ is its own commensurator if and only if $X^{0}=Y^{\infty}$.
\end{abstract}

\section{INTRODUCTION}

Let $S$ be a finite set. A Coxeter matrix over $S$ is a matrix $M=\left(m_{s, t}\right)_{s, t \in S}$ indexed by the elements of $S$ and satisfying

(a) $m_{s, s}=1$ if $s \in S$,

(b) $m_{s, t}=m_{t, s} \in\{2,3,4, \ldots,+\infty\}$ if $s, t \in S$ and $s \neq t$.

A Coxeter matrix $M=\left(m_{s, t}\right)_{s, t \in S}$ is usually represented by its Coxeter graph $\Gamma$. This is defined by the following data.

(a) $S$ is the set of vertices of $\Gamma$.

(b) Two vertices $s, t \in S$ are joined by an edge if $m_{s, t} \geq 3$.

(c) The edge joining two vertices $s, t \in S$ is labeled by $m_{s, t}$ if $m_{s, t} \geq 4$.

The Coxeter system associated with $M$ (or with $\Gamma$ ) is the pair $(W, S)$ where $W$ is the group having the presentation

$$
\left.W=\langle S|(s t)^{m_{s, t}}=1 \text { if } m_{s, t}<+\infty\right\rangle .
$$

The group $W$ is called the Coxeter group associated with $M$. Given $X \subseteq S$, we write

$$
M_{X}=\left(m_{s, t}\right)_{s, t \in X},
$$

$\Gamma_{X}$ the Coxeter graph which represents $M_{X}$,

$W_{X}$ the subgroup of $W$ generated by $X$.

Received by the editors October 17, 1995.

1991 Mathematics Subject Classification. Primary 20 F55.

(C)1997 American Mathematical Society 
The pair $\left(W_{X}, X\right)$ is the Coxeter system associated with $M_{X}$ (see [Bo, Ch. IV, $\S 1$, $\left.n^{\circ} 8\right]$ ). The group $W_{X}$ is called a parabolic subgroup of the Coxeter system $(W, S)$. We assume that the reader is familiar with the theory of Coxeter groups. We refer to $[\mathrm{Bo}]$ and $[\mathrm{Hu}]$ for general expositions on the subject.

For a group $G$ and for a subgroup $H$ of $G$, we denote by $Z(G)$ the center of $G$, by $Z_{G}(H)$ the centralizer of $H$ in $G$, by $N_{G}(H)$ the normalizer of $H$ in $G$, and by $C_{G}(H)$ the commensurator of $H$ in $G$. Recall that this is defined by

$$
C_{G}(H)=\left\{g \in G ; H \cap\left(g H g^{-1}\right) \text { has finite index in both } H \text { and } g H^{-1}\right\} \text {. }
$$

Commensurators play an important role in representation theory, especially in the study of induced representations. For example, if a subgroup $H$ of $G$ is its own commensurator, then any finite dimensional irreducible representation of $H$ induces an irreducible representation of $G$ (see [Ma]). If $\mathbf{K}$ is an infinite field and $P$ is a parabolic subgroup of $\mathrm{GL}(n, \mathbf{K})$, then $P$ is its own commensurator (see $[\mathrm{BH}]$ ). A similar result is obviously not true for Coxeter groups. Indeed, the commensurator of a finite parabolic subgroup is the whole group $W$. However, we prove in this paper that the commensurator of a parabolic subgroup is always a parabolic subgroup (Corollary 2.2), and we give a criterion which decides whether a parabolic subgroup is its own commensurator (Corollary 2.3).

The goal of this paper is to determine the commensurator of a parabolic subgroup $W_{X}$ of a Coxeter system. This subgroup can be decomposed in the form $W_{X}=W_{X^{0}} \cdot W_{X^{\infty}} \simeq W_{X^{0}} \times W_{X^{\infty}}$, where $W_{X^{0}}$ is finite and all the irreducible components of $W_{X^{\infty}}$ are infinite. In a first step (Proposition 2.4), we prove that the commensurator of $W_{X}$ is the normalizer of $W_{X^{\infty}}$. In a second step (Proposition $2.5)$, we prove that the normalizer of $W_{X^{\infty}}$ is $Q Z_{W}\left(W_{X^{\infty}}\right) \cdot W_{X^{\infty}}$, where

$$
Q Z_{W}\left(W_{X^{\infty}}\right)=\left\{w \in W ; w X^{\infty} w^{-1}=X^{\infty}\right\}
$$

is the quasi-centralizer of $W_{X^{\infty}}$. In a third step (Proposition 2.6), we prove that $Q Z_{W}\left(W_{X^{\infty}}\right)$ is $W_{Y^{\infty}}$, where $Y^{\infty}$ is the set of $t$ in $S$ such that $m_{s, t}=2$ for all $s \in X^{\infty}$. Finally, from Propositions 2.4, 2.5 and 2.6, we deduce the following expression of the commensurator of $W_{X}$ (Theorem 2.1):

$$
C_{W}\left(W_{X}\right)=W_{Y^{\infty}} \cdot W_{X^{\infty}} \simeq W_{Y^{\infty}} \times W_{X^{\infty}} .
$$

We state our results precisely in Section 2, and we prove them in Section 3.

\section{Statements}

From now on, we fix a Coxeter system $(W, S)$.

Let $X$ be a subset of $S$. Let $\Gamma_{1}, \ldots, \Gamma_{n}$ be the connected components of $\Gamma_{X}$ and, for $i \in\{1, \ldots, n\}$, let $X_{i}$ be the set of vertices of $\Gamma_{i}$. The group $W_{X_{i}}$ is called an irreducible component of $W_{X}$. It is clear that

$$
W_{X}=W_{X_{1}} \cdots W_{X_{n}} \simeq W_{X_{1}} \times \cdots \times W_{X_{n}} .
$$

We assume that $W_{X_{i}}$ is finite if $i=1, \ldots, r$, and that $W_{X_{i}}$ is infinite if $i=r+$ $1, \ldots, n$. We set

$$
\begin{aligned}
& X^{0}=X_{1} \cup \cdots \cup X_{r}, \\
& X^{\infty}=X_{r+1} \cup \cdots \cup X_{n} .
\end{aligned}
$$

Then

$$
W_{X}=W_{X^{0}} \cdot W_{X^{\infty}} \simeq W_{X^{0}} \times W_{X^{\infty}},
$$


the group $W_{X^{0}}$ is finite, and all the irreducible components of $W_{X^{\infty}}$ are infinite.

Theorem 2.1. Let $X$ be a subset of $S$. Then

$$
C_{W}\left(W_{X}\right)=W_{Y^{\infty}} \cdot W_{X^{\infty}}=W_{Y^{\infty} \cup X^{\infty}} \simeq W_{Y^{\infty}} \times W_{X^{\infty}},
$$

where

$$
Y^{\infty}=\left\{t \in S ; m_{s, t}=2 \text { for all } s \in X^{\infty}\right\} .
$$

Corollary 2.2. The commensurator of a parabolic subgroup of $(W, S)$ is a parabolic subgroup.

Corollary 2.3. Let $X$ be a subset of $S$. Then $W_{X}$ is its own commensurator if and only if $X^{0}$ is the set of $t \in S$ such that $m_{s, t}=2$ for all $s \in X^{\infty}$.

Theorem 2.1 is a direct consequence of the following Propositions 2.4, 2.5, and 2.6 .

Proposition 2.4. Let $X$ be a subset of $S$. Then the commensurator of $W_{X}$ in $W$ is equal to the normalizer of $W_{X^{\infty}}$ in $W$.

We define the quasi-center of $(W, S)$ to be

$$
Q Z(W, S)=\left\{w \in W ; w S w^{-1}=S\right\} .
$$

Similarly, we define the quasi-centralizer of a parabolic subgroup $W_{X}$ of $(W, S)$ to be

$$
Q Z_{W}\left(W_{X}\right)=\left\{w \in W ; w X w^{-1}=X\right\} .
$$

Proposition 2.5. Let $X$ be a subset of $S$. Then

$$
N_{W}\left(W_{X}\right)=Q Z_{W}\left(W_{X}\right) \cdot W_{X} .
$$

Moreover,

$$
Q Z_{W}\left(W_{X}\right) \cap W_{X}=Q Z\left(W_{X}, X\right) .
$$

Proposition 2.6. Let $X$ be a subset of $S$ such that all the irreducible components of $W_{X}$ are infinite (i.e. $X=X^{\infty}$ ). Let

$$
Y=\left\{t \in S ; m_{s, t}=2 \text { for all } s \in X\right\} .
$$

Then the quasi-centralizer of $W_{X}$ is equal to $W_{Y}$.

Proposition 2.4 is a consequence of [So, Lemma 2]. Proposition 2.5 is stated in [Ho] for finite type Coxeter systems (see also [Kr, Ch. 3]). Moreover, its proof is quite simple. Proposition 2.6 is a consequence of [De, Prop. 5.5].

\section{Proofs}

First, we state in Lemmas 3.1 and 3.2 some well-known facts that will be required later. Recall that each $w \in W$ can be written $w=s_{1} \ldots s_{r}$ where $s_{i} \in S$ for all $i \in\{1, \ldots, r\}$. If $r$ is as small as possible, then $r$ is called the length of $w$ and is denoted by $l(w)$.

Lemma 3.1 (Bourbaki [Bo, Ch. IV, $\S 1$, Ex. 3]). Let $X$ and $X^{\prime}$ be two subsets of $S$. 
(i) Let $w \in W$. There is a unique element $v$ of minimal length in $W_{X} w W_{X^{\prime}}$. Moreover, each $w^{\prime} \in W_{X} w W_{X^{\prime}}$ can be written as $w^{\prime}=u v u^{\prime}$, where $u \in W_{X}$, $u^{\prime} \in W_{X^{\prime}}$, and $l\left(w^{\prime}\right)=l(u)+l(v)+l\left(u^{\prime}\right)$. An element $v$ is called $\left(X, X^{\prime}\right)$ reduced if it is of minimal length in $W_{X} v W_{X^{\prime}}$.

(ii) If an element $v$ is $(X, \emptyset)$-reduced, then $l(u v)=l(u)+l(v)$ for all $u \in W_{X}$.

(iii) If an element $v$ is $\left(\emptyset, X^{\prime}\right)$-reduced, then $l\left(v u^{\prime}\right)=l(v)+l\left(u^{\prime}\right)$ for all $u^{\prime} \in W_{X^{\prime}}$.

(iv) An element $v$ is $(X, \emptyset)$-reduced if and only if $l(s v)>l(v)$ for all $s \in X$.

(v) An element $v$ is $\left(\emptyset, X^{\prime}\right)$-reduced if and only if $l\left(v s^{\prime}\right)>l(v)$ for all $s^{\prime} \in X^{\prime}$.

(vi) An element $v$ is $\left(X, X^{\prime}\right)$-reduced if and only if it is both $(X, \emptyset)$-reduced and $\left(\emptyset, X^{\prime}\right)$-reduced.

Lemma 3.2 (Bourbaki [Bo, Ch. IV, $§ 1$, Ex. 22]). Let $w_{0}$ be an element of $W$. The following statements are equivalent.

(1) $l\left(s w_{0}\right)<l\left(w_{0}\right)$ for all $s \in S$.

(2) $l\left(w_{0} s\right)<l\left(w_{0}\right)$ for all $s \in S$.

(3) $l\left(w w_{0}\right)=l\left(w_{0}\right)-l(w)$ for all $w \in W$.

(4) $l\left(w_{0} w\right)=l\left(w_{0}\right)-l(w)$ for all $w \in W$.

Such an element is unique and exists if and only if $W$ is finite. Then it is the unique element of maximal length in $W$. Moreover, $w_{0}^{2}=1$ and $w_{0} S w_{0}=S$.

The following proposition is the key of the proof of Proposition 2.4.

Proposition 3.3 (Solomon [So, Lemma 2]). Let $X$ and $X^{\prime}$ be two subsets of $S$, and let $v$ be $a\left(X, X^{\prime}\right)$-reduced element of $W$. Then

$$
W_{X} \cap\left(v W_{X^{\prime}} v^{-1}\right)=W_{Y},
$$

where $Y=\left(v X^{\prime} v^{-1}\right) \cap X$.

Corollary 3.4. Let $X$ and $X^{\prime}$ be two subsets of $S$, and let $w$ be an element of $W$. We write $w=u_{0} v u_{0}^{\prime}$, where $u_{0} \in W_{X}, u_{0}^{\prime} \in W_{X^{\prime}}$, and $v$ is $\left(X, X^{\prime}\right)$-reduced. Then

$$
W_{X} \cap\left(w W_{X^{\prime}} w^{-1}\right)=u_{0} W_{Y} u_{0}^{-1},
$$

where $Y=\left(v X^{\prime} v^{-1}\right) \cap X$.

Proof.

$$
\begin{aligned}
W_{X} \cap\left(w W_{X^{\prime}} w^{-1}\right) & =W_{X} \cap\left(u_{0} v u_{0}^{\prime} W_{X^{\prime}} u_{0}^{\prime-1} v^{-1} u_{0}^{-1}\right) \\
& =W_{X} \cap\left(u_{0} v W_{X^{\prime}} v^{-1} u_{0}^{-1}\right) \\
& =u_{0}\left(\left(u_{0}^{-1} W_{X} u_{0}\right) \cap\left(v W_{X^{\prime}} v^{-1}\right)\right) u_{0}^{-1} \\
& =u_{0}\left(W_{X} \cap\left(v W_{X^{\prime}} v^{-1}\right)\right) u_{0}^{-1} \\
& =u_{0} W_{Y} u_{0}^{-1} .
\end{aligned}
$$

Proof of Proposition 2.4. Let $w \in N_{W}\left(W_{X^{\infty}}\right)$. Then

$$
W_{X^{\infty}}=w W_{X^{\infty}} w^{-1} \subseteq W_{X} \cap\left(w W_{X} w^{-1}\right),
$$

the group $W_{X \infty}$ has finite index in $W_{X}$, and the group $w W_{X \infty} w^{-1}$ has finite index in $w W_{X} w^{-1}$. Thus $W_{X} \cap\left(w W_{X} w^{-1}\right)$ has finite index in both $W_{X}$ and $w W_{X} w^{-1}$. This shows that $N_{W}\left(W_{X^{\infty}}\right) \subseteq C_{W}\left(W_{X}\right)$. 
Let $w \in C_{W}\left(W_{X}\right)$. We write $w=u_{0} v u_{0}^{\prime}$, where $u_{0}, u_{0}^{\prime} \in W_{X}$ and $v$ is $(X, X)$ reduced. By Corollary 3.4,

$$
W_{X} \cap\left(w W_{X} w^{-1}\right)=u_{0} W_{Y} u_{0}^{-1},
$$

where $Y=\left(v X v^{-1}\right) \cap X$. Let $Y^{0}=Y \cap X^{0}$, and let $Y^{\infty}=Y \cap X^{\infty}$. For a group $G$ and for a subgroup $H$ of $G$, we denote by $|G: H|$ the index of $H$ in $G$. Then

$$
\begin{gathered}
\left|W_{X}: W_{X} \cap\left(w W_{X} w^{-1}\right)\right|=\left|W_{X}: u_{0} W_{Y} u_{0}^{-1}\right|=\left|W_{X}: W_{Y}\right| \\
=\left|W_{X^{0}}: W_{Y^{0}}\right| \cdot\left|W_{X^{\infty}}: W_{Y^{\infty}}\right| .
\end{gathered}
$$

If $Y^{\infty} \neq X^{\infty}$, then, by [De, Prop. 4.2], $W_{Y^{\infty}}$ has infinite index in $W_{X^{\infty}}$; thus $W_{X} \cap\left(w W_{X} w^{-1}\right)$ has infinite index in $W_{X}$, too. This is not the case; thus $Y^{\infty}=$ $X^{\infty}$. Let $\Gamma_{1}, \ldots, \Gamma_{n}$ be the connected components of $\Gamma_{X}$, and, for $i=1, \ldots, n$, let $X_{i}$ be the set of vertices of $\Gamma_{i}$. We assume that $X^{0}=X_{1} \cup \cdots \cup X_{r}$ and that $X^{\infty}=X_{r+1} \cup \cdots \cup X_{n}$. Let $i \in\{r+1, \ldots, n\}$. Then

$$
v^{-1} X_{i} v \subseteq v^{-1} X^{\infty} v=v^{-1} Y^{\infty} v \subseteq v^{-1} Y v \subseteq X
$$

Thus there exists $j \in\{1, \ldots, r, r+1, \ldots, n\}$ such that $v^{-1} X_{i} v \subseteq X_{j}$. The group $W_{X_{i}}$ is infinite and $v^{-1} W_{X_{i}} v \subseteq W_{X_{j}}$; thus $W_{X_{j}}$ is infinite, and so $j \in\{r+1, \ldots, n\}$. This shows that $v^{-1} X^{\infty} v \subseteq X^{\infty}$; thus $v X^{\infty} v^{-1}=X^{\infty}$; therefore $v W_{X \infty} v^{-1}=$ $W_{X^{\infty}}$. On the other hand, since $W_{X}=W_{X^{0}} \cdot W_{X^{\infty}} \simeq W_{X^{0}} \times W_{X^{\infty}}$, we have $u W_{X} \infty u^{-1}=W_{X \infty}$ for all $u \in W_{X}$. So,

$w W_{X \infty} w^{-1}=u_{0} v u_{0}^{\prime} W_{X \infty} u_{0}^{\prime-1} v^{-1} u_{0}^{-1}=u_{0} v W_{X^{\infty}} v^{-1} u_{0}^{-1}=u_{0} W_{X} \infty u_{0}^{-1}=W_{X^{\infty}}$.

This shows that $C_{W}\left(W_{X}\right) \subseteq N_{W}\left(W_{X^{\infty}}\right)$.

Proof of Proposition 2.5. The inclusion

$$
Q Z_{W}\left(W_{X}\right) \cdot W_{X} \subseteq N_{W}\left(W_{X}\right)
$$

is obvious.

Let $w \in N_{W}\left(W_{X}\right)$. We write $w=v u$, where $u \in W_{X}, v$ is $(\emptyset, X)$-reduced, and $l(w)=l(v)+l(u)$. We have

$$
w W_{X} w^{-1}=v W_{X} v^{-1}=W_{X} .
$$

The element $v$ is of minimal length in $v W_{X}=W_{X} v$; thus $v$ is also $(X, \emptyset)$-reduced. If $s \in X$, then, by Lemma 3.1,

$$
\begin{aligned}
& l(v)+1=l(v s)=l\left(v s v^{-1} v\right)=l\left(v s v^{-1}\right)+l(v) \\
\Rightarrow & l\left(v s v^{-1}\right)=1 \\
\Rightarrow & v s v^{-1} \in W_{X} \cap S=X .
\end{aligned}
$$

So, $v X v^{-1} \subseteq X$; thus $v X v^{-1}=X$; therefore $v \in Q Z_{W}\left(W_{X}\right)$. This shows that $N_{W}\left(W_{X}\right) \subseteq Q Z_{W}\left(W_{X}\right) \cdot W_{X}$.

The equality

$$
Q Z_{W}\left(W_{X}\right) \cap W_{X}=Q Z\left(W_{X}, X\right)
$$

is obvious. 
Before proving Proposition 2.6, we recall some facts on root systems. Let $V$ be a real vector space having a basis $\left\{e_{s} ; s \in S\right\}$ in one-to-one correspondence with $S$. Let $B$ be the symmetric bilinear form on $V$ defined by

$$
B\left(e_{s}, e_{t}\right)=\left\{\begin{array}{l}
-\cos \left(\pi / m_{s, t}\right) \quad \text { if } m_{s, t}<+\infty \\
-1 \quad \text { if } m_{s, t}=+\infty
\end{array}\right.
$$

There is an action of $W$ on $V$ defined by

$$
s(x)=x-2 B\left(x, e_{s}\right) e_{s}
$$

if $s \in S$ and $x \in V$. This action is called the canonical representation of $(W, S)$. The root system $\Phi$ of $(W, S)$ is the collection of all vectors $w\left(e_{s}\right)$ where $w \in W$ and $s \in S$. By [Bo, Ch. V, $\S 4$, Ex. 8], every root $\alpha$ can be uniquely written in the form

$$
\alpha=\sum_{s \in S} a_{s} e_{s} \quad\left(a_{s} \in \mathbf{R}\right)
$$

where either all $a_{s}$ are positive, or all $a_{s}$ are negative. We call $\alpha$ positive and write $\alpha>0$ if $a_{s} \geq 0$ for all $s \in S$. We call $\alpha$ negative and write $\alpha<0$ if $a_{s} \leq 0$ for all $s \in S$.

Proposition 3.5 (Deodhar [De, Prop. 3.1]). Let

$$
T=\left\{w s w^{-1} ; w \in W \text { and } s \in S\right\},
$$

and let $\Phi^{+}$be the set of positive roots. For $\alpha=w\left(e_{s}\right)$, we write $r_{\alpha}=w s w^{-1}$. Then the function $\Phi^{+} \rightarrow T\left(\alpha \mapsto r_{\alpha}\right)$ is well-defined and bijective.

Proposition 3.6 (Deodhar [De, Prop. 2.2]). Let $w \in W$, and let $s \in S$. Then $l(w s)>l(w)$ if and only if $w\left(e_{s}\right)>0$.

For a subset $X$ of $S$, we write

$$
E_{X}=\left\{e_{s} ; s \in X\right\}
$$

The following lemma is an easy consequence of Propositions 3.5 and 3.6.

Lemma 3.7. Let $X$ and $X^{\prime}$ be two subsets of $S$, and let $w$ be an element of $W$. The following statements are equivalent.

(1) $w\left(E_{X}\right)=E_{X^{\prime}}$.

(2) $w X w^{-1}=X^{\prime}$ and $l(w s)>l(w)$ for all $s \in X$.

For $X \subseteq S$ such that $W_{X}$ is finite, we denote by $w_{X}$ the unique element of maximal length in $W_{X}$.

Let $X$ be a subset of $S$, and let $t$ be an element of $S \backslash X$. Let $\Gamma_{0}$ be the connected component of $\Gamma_{\{t\} \cup X}$ containing $t$, and let $Y_{0}$ be the set of vertices of $\Gamma_{0}$. We say that $t$ is $X$-admissible if $W_{Y_{0}}$ is finite. In that case, we write

$$
c(t, X)=w_{Y_{0}} w_{X_{0}},
$$

where $X_{0}=Y_{0} \backslash\{t\}$. It is the element of minimal length in $w_{Y_{0}} W_{X}$. In particular, $c(t, X)$ is $(\emptyset, X)$-reduced. By Lemma 3.2 and Lemma 3.7, there exists a subset $X^{\prime}$ of $\{t\} \cup X$ such that

$$
c(t, X)\left(E_{X}\right)=E_{X^{\prime}}
$$

If $X=X^{\infty}$, then $t$ is $X$-admissible if and only if $m_{s, t}=2$ for all $s \in X$. In that case, $c(t, X)=t$ and $c(t, X)\left(E_{X}\right)=E_{X}$. 
Proposition 3.8 (Deodhar [De, Prop. 5.5]). Let $X$ and $X^{\prime}$ be two subsets of $S$, and let $w$ be an element of $W$. If $w\left(E_{X}\right)=E_{X^{\prime}}$, then there exist sequences

$X_{0}=X, X_{1}, \ldots, X_{n}=X^{\prime}$ of subsets of $S$, $t_{0}, t_{1}, \ldots, t_{n-1}$ of elements of $S$,

such that

(a) $t_{i} \in S \backslash X_{i}$ and $t_{i}$ is $X_{i}$-admissible $(i=0,1, \ldots, n-1)$,

(b) $c\left(t_{i}, X_{i}\right)\left(E_{X_{i}}\right)=E_{X_{i+1}}(i=0,1, \ldots, n-1)$,

(c) $w=c\left(t_{n-1}, X_{n-1}\right) \ldots c\left(t_{1}, X_{1}\right) c\left(t_{0}, X_{0}\right)$.

The following Lemmas 3.9 and 3.10 are preliminary results to the proof of Proposition 2.6 .

Lemma 3.9. Let $X$ and $X^{\prime}$ be two subsets of $S$, and let $w$ be an element of $W$. If $w X w^{-1}=X^{\prime}$, then $w$ can be written $w=v u$, where $u \in Q Z\left(W_{X}, X\right), v X v^{-1}=X^{\prime}$, and $l(v s)>l(v)$ for all $s \in X$.

Proof. We write $w=v u$, where $u \in W_{X}, v$ is $(\emptyset, X)$-reduced, and $l(w)=l(v)+l(u)$. We have

$$
w W_{X} w^{-1}=v W_{X} v^{-1}=W_{X^{\prime}} .
$$

The element $v$ is of minimal length in $v W_{X}=W_{X^{\prime}} v$; thus $v$ is also $\left(X^{\prime}, \emptyset\right)$-reduced. If $s \in X$, then, by Lemma 3.1,

$$
\begin{aligned}
& l(v)+1=l(v s)=l\left(v s v^{-1} v\right)=l\left(v s v^{-1}\right)+l(v) \\
\Rightarrow & l\left(v s v^{-1}\right)=1 \\
\Rightarrow & v s v^{-1} \in W_{X^{\prime}} \cap S=X^{\prime} .
\end{aligned}
$$

So, $v X v^{-1} \subseteq X^{\prime}$. Similarly, $v^{-1} X^{\prime} v \subseteq X$. Thus $v X v^{-1}=X^{\prime}$.

Since $v$ is $(\emptyset, X)$-reduced, by Lemma 3.1, $l(v s)>l(v)$ for all $s \in X$.

Finally,

$$
\begin{aligned}
& w X w^{-1}=v u X u^{-1} v^{-1}=X^{\prime} \\
\Rightarrow & u X u^{-1}=v^{-1} X^{\prime} v=X .
\end{aligned}
$$

Thus $u \in Q Z\left(W_{X}, X\right)$.

Lemma 3.10 (Bourbaki $[$ Bo, Ch. V, $\S 4$, Ex. 3]). We suppose that $(W, S)$ is irreducible.

(i) If $W$ is finite, then $Q Z(W, S)=\left\{1, w_{0}\right\}$, where $w_{0}$ is the unique element of maximal length in $W$.

(ii) If $W$ is infinite, then $Q Z(W, S)=\{1\}$.

Proof of Proposition 2.6. The inclusion

$$
W_{Y} \subseteq Q Z_{W}\left(W_{X}\right)
$$

is obvious.

Let $w \in Q Z_{W}\left(W_{X}\right)$. By Lemma 3.9, $w$ can be written $w=v u$, where $u \in$ $Q Z\left(W_{X}, X\right), v X v^{-1}=X$, and $l(v s)>l(v)$ for all $s \in X$. Since $X=X^{\infty}$, by Lemma 3.10, $Q Z\left(W_{X}, X\right)=\{1\}$; thus $u=1$. By Lemma 3.7, $v\left(E_{X}\right)=E_{X}$. By Proposition 3.8, there exist sequences

$X=X_{0}, X_{1}, \ldots, X_{n}=X$ of subsets of $S$, $t_{0}, t_{1}, \ldots, t_{n-1}$ of elements of $S$, 
such that

(a) $t_{i} \in S \backslash X_{i}$ and $t_{i}$ is $X_{i}$-admissible $(i=0,1, \ldots, n-1)$,

(b) $c\left(t_{i}, X_{i}\right)\left(E_{X_{i}}\right)=E_{X_{i+1}}(i=0,1, \ldots, n-1)$,

(c) $v=c\left(t_{n-1}, X_{n-1}\right) \ldots c\left(t_{1}, X_{1}\right) c\left(t_{0}, X_{0}\right)$.

Since $X=X^{\infty}$, if $X_{i}=X$, then $m_{t_{i}, s}=2$ for all $s \in X$ (namely, $t_{i} \in Y$ ), $c\left(t_{i}, X_{i}\right)=t_{i}$, and $X_{i+1}=X$ (since $\left.t_{i}\left(E_{X}\right)=E_{X}\right)$. Since $X_{0}=X$, it follows that $c\left(t_{i}, X_{i}\right)=t_{i} \in Y$ for all $i=0,1, \ldots, n-1$. Thus

$$
w=v=t_{n-1} \ldots t_{1} t_{0} \in W_{Y} .
$$

This shows that $Q Z_{W}\left(W_{X}\right) \subseteq W_{Y}$.

\section{REFERENCES}

[Bo] N. Bourbaki, "Groupes et algèbres de Lie, Chapitres IV-VI", Hermann, Paris, 1968. MR 39: 1590

[Br] K. S. Brown, "Buildings", Springer-Verlag, New York, 1989. MR 90e:20001

[BH] M. Burger and P. de la Harpe, Irreducible representations of discrete groups, in preparation.

[De] V. V. Deodhar, On the root system of a Coxeter group, Comm. Algebra 10 (1982), 611-630. MR 83j:20052a

[Ho] R. B. Howlett, Normalizers of parabolic subgroups of reflection groups, J. London Math. Soc. (2) 21 (1980), 62-80. MR 81g:20094

$[\mathrm{Hu}]$ J. E. Humphreys, "Reflection groups and Coxeter groups", Cambridge studies in advanced mathematics, vol. 29, Cambridge University Press, 1990. MR 92h:20002

[Kr] D. Krammer, "The conjugacy problem for Coxeter groups", Ph. D. Thesis, Utrecht, 1994.

[Ma] G. W. Mackey, "The theory of unitary group representations", The University of Chicago Press, 1976. MR 53:686

[So] L. Solomon, A Mackey formula in the group ring of a Coxeter group, J. Algebra 41 (1976), 255-264. MR 56:3104

Laboratoire de Topologie, Département de Mathématiques, Université de BourGogne, U.M.R. 5584, B.P. 138, 21004 Dijon Cedex, France

E-mail address: lparis@satie.u-bourgogne.fr 